\title{
The magmaFreiburg Soccer Team
}

\author{
Klaus Dorer \\ Centre for Cognitive Science \\ Institute for Computer Science and Social Research \\ Albert-Ludwigs-University Freiburg, Germany \\ klaus@cognition.iig.uni-freiburg.de
}

\section{Introduction}

The main interest of our research concerns motivation action control and goal management of agents (magma). Action Control of the magmaFreiburg team is based on extended behavior networks, which add situation-dependent motivational influences to the agent, extend original behavior networks to exploit information from continuous domains and allow concurrent execution of behaviors. Advantages of the original networks, such as reactivity, planning capabilities, consideration of multiple goals and its cheap calculations are maintained.

magmaFreiburg has been very successful in the competition finishing at second place. We scored 59:0 goals and 12:0 points in the four games of the round robin and 30:11 goals in the six games of the elimination round with all goals against us scored by the winning team CMUnited.

\section{Team Development}

Team Leader: Klaus Dorer

Team Members:

Markus Plewinski, Marc Haas

- Fachhochschule Furtwangen

- Germany

- students

- did not attend the competition

Web page http://www.iig.uni-freiburg.de/cognition/members/klaus /robocup/magmaFreiburg.html

\section{World Model}

Each time an agent receives a perception from the server the information is entered into a local map containing the distances and directions of visible objects. After self-localization, the global position and direction of the agent and all visible objects are inserted into a global map. Information of non-visible objects gained by communication (see next section) is also entered into the map. The information in the map is updated before action selection, taking into account 
information on the expected effects of the agents previous actions and the inertia of the ball and the agent. Moveable objects like other players and the ball are removed from the map after three seconds or if they are not seen at the expected position. Functional objects are calculated from the information in the global map, using indexical-functional aspects of the situation [1]. This reduces the number of competence modules needed (see section 7 ).

\section{Communication}

Communication is used by the magmaFreiburg agents to share information on visible objects and the agent's internal information. This allows agents to improve information on visible players and to know about the position of players the agent can not see. Knowledge about the stamina of other agents allow players to replace tired teammates. To coordinate communication between agents, a locker-room agreement is used [6].

\section{Skills}

Players anticipate the future positions (20 cycles) of the ball with respect to its current velocity. They calculate the possible intersection point by taking into account the number of cycles the agent needs to approach the corresponding ball position. When receiving a ball, the agent takes into account the ball's speed to calculate the proper power vector for kicking. If the velocity of the ball is low and the agent decides to do a hard kick, the agent tries to place the ball in a position, where it can kick the ball twice. Due to poor dribbling abilities, the ball is lost in one third of all attempts. The goalie always keeps the ball in view. This is done by turning the neck (as other agents do) if moving sideways to the ball and by moving backwards if moving away from the ball. The goalie also tries to position itself in a way to minimize the attacker's angle to the goal.

\section{$6 \quad$ Strategy}

The player with the ball has choice of four behaviors: it can dribble with the ball, pass the ball to a teammate, kick towards the goal or just clear the ball in any direction. The mechanism used for behavior selection is described in the next section. Movement of defenders and midfielders without the ball is restricted to moving forward in order to create an offside trap if the ball is in front of the offside line, and moving backward if the ball is behind them. Midfielders and offenders without the ball try to keep themselves onside and try to keep at the level of the ball within the opponents half when attacking.

\section{Special Team Features}

Behavior selection of our agents is controlled by extended behavior networks [2, $3]$ that are based on work by Maes $[4,5]$. Extended behavior networks consist 


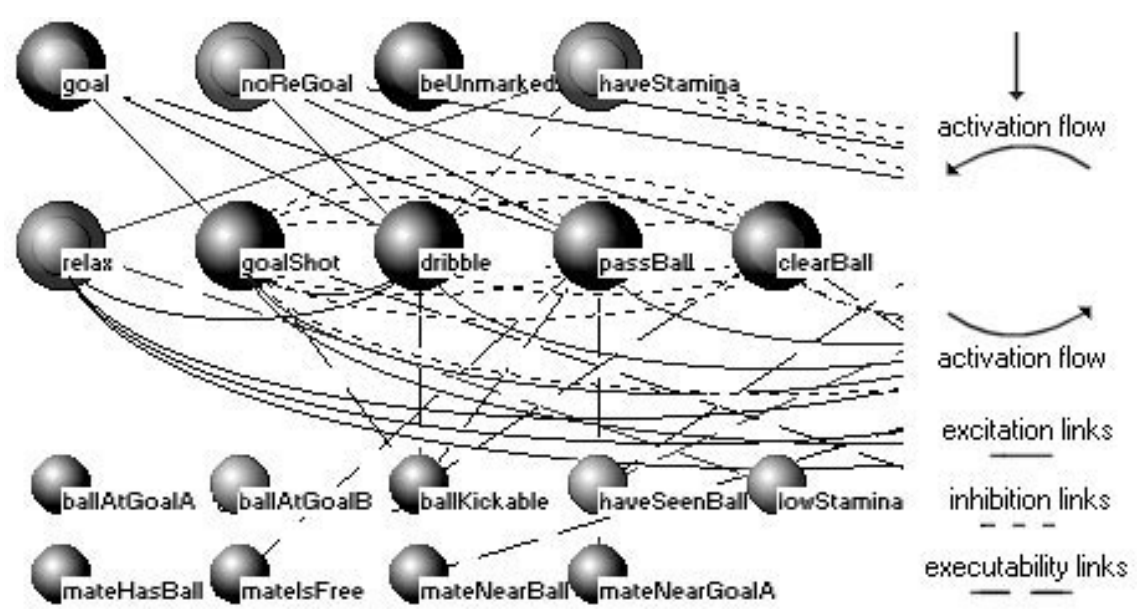

Fig. 1. Part of a behavior network used by a soccer-agent. The goals of the agent are at the top level, in the middle the competence modules and at the bottom level the situation propositions (perceptions). (The complete network contains 14 competences).

of the goals of an agent, a set of behavior rules called competence modules, the perceptions of the agent and resource nodes (see fig. 1).

Goals represent the utility of propositions that are part of the goal condition. Goals can be statically prioritized by their importance and can be dynamically, i.e. situation-dependent, prioritized by their relevance condition. The utility of a goal is calculated as the product of its importance and its relevance.

A competence module consists of the preconditions that have to be satisfied for the module to be executable, a corresponding behavior, the effects expected after behavior execution, the resources used by the behavior and an activation value. The activation of a competence module can be interpreted as the expected utility of the module's behavior with $e u=\sum_{i} a_{i} \cdot e x_{i}$, where $a_{i}$ is the utility of effect $i$ and $e x_{i}$ is the probability of effect $i$ to become true. The utility of effects that are part of a goal condition can be directly accessed by links from the goal to the competence module. The utility of propositions that are not part of a goal condition can be calculated by utility propagation using links between competence modules. Any unsatisfied proposition of a precondition is assigned a utility corresponding to the expected utility of the competence module. Preconditions of competence modules with a high expected utility get important subgoals of the network. For a more detailed description on utility propagation see [2]. The execution of a competence module depends on its executability, the expected utility and the availability of needed resources. Modules with high expected utility are preferred.

Perceptions represent the truth values of propositions in a domain. To improve the quality of perception within continuous domains, real-valued propositions have been introduced by extended behavior networks. This has implications on the executability of competence modules, which becomes real-valued, and on 
the relevance of goals, which can have continuous values. Empirical results show that real-valued propositions improve the quality of behavior selection in the RoboCup domain [2].

Resource nodes are used to coordinate the selection of multiple concurrent behaviors. Competence modules are connected with the resource nodes that correspond to the resources they use. Using these links a competence module can make sure that enough resources are available to execute the corresponding behavior and that it is the module with the highest utility requesting the resource. Concurrent actions like speaking, turning the neck and dashing have been realized by this domain independent mechanism for concurrent behavior selection.

Behavior selection in extended behavior networks is extremely cheap to calculate. All eleven agents of the magmaFreiburg team have been run on a single PC while other teams used up to five PCs. Since behavior selection can be calculated locally in each competence module, calculations can be done in parallel and could even be improved if each node of the network were run on its own processor. Besides being reactive, extended behavior networks also prefer goaldirected behavior by calculating the expected utility of a behavior with respect to the goals. In contrast to purely reactive approaches, goals of an agent can be explicitly specified.

\section{Conclusion}

The success in RoboCup99 has encouraged our team to take part in Melbourne 2000. Besides improvements to existing behaviors (especially ball handling and dribbling), we plan to add new behaviors to improve positioning of players not dealing with the ball and to introduce situation-dependent team strategies.

\section{References}

1. Agre, Ph., and Chapman, D. (1987). Pengi: An Implementation of a Theory of Activity. In: Proceedings of the Sixth National Conference on Artificial Intelligence, AAAI-87, Morgan Kaufmann, Los Altos.

2. Dorer, K. (1999). Behavior Networks for Continous Domains using SituationDependent Motivations. Proceedings of the $16^{\text {th }}$ International Joint Conference on Artificial Intelligence, pages 1233-1238, Morgan Kaufmann, San Francisco.

3. Dorer, K. (1999). Extended Behavior Networks for the magmaFreiburg Soccer Team. In: S. Coradeschi, T. Balch, G. Kraetzschmar and P. Stone, Team Descriptions Simulation League, Linköping University Electronic Press, Stockholm.

4. Maes, P. (1989). The Dynamics of Action Selection. In Proceedings of the International Joint Conference on Artificial Intelligence-'89, Morgan Kaufmann, Detroit.

5. Maes, P. (1990). Situated Agents Can Have Goals. In Journal for Robotics and Autonomous Systems, Vol. 6, No 1, pages 49-70, North-Holland.

6. P. Stone, M. Veloso, und P. Riley. (1999). The cmunited-98 champion simulator team. In M. Asada und H. Kitano, editors, RoboCup-98: Robot Soccer World Cup II. Springer, Berlin. 